\title{
A closed dimensionless linear set
}

\author{
By E. Best.
}

(Received 13th June, 1939. Read 3rd November, 1939.)

1. The problem which we discuss in this paper can be easily settled for a closed plane set; after a brief introduction giving the definitions and theorems used later we indicate how this may be done; we then proceed to the main problem.

2. We are concerned with the measure and dimension theory of sets of points formulated by Hausdorfi'. Let $A$ be any set of points in a Euclidean space of $n$ dimensions ( $n$ any integer); and let $\lambda(t)$ be any function of the real variable $t$, defined in some interval $0 \leqq t \leqq t_{0}$ in which it is continuous, concave and strictly increasing, and such that $\lambda(0)=0$. Given a positive number $\rho \leqq t_{0}$, denote by $U(A, \rho)$ any set of spheres $\left\{U_{r}\right\}$ of respective diameters $\left\{d_{r}\right\}$ such that (i) $d_{r}<\rho$ for each $r$, and (ii) each point of $A$ is interior to at least one sphere $U_{r}$. Then

$$
\text { lower bound } \sum_{U(A, \rho)} \lambda\left(d_{r}\right)
$$

is denoted by $\lambda-m_{\rho} A$, and $\lim _{\rho \rightarrow 0}\left(\lambda-m_{\rho} A\right)$ (which obviously exists) is denoted by $\lambda-m^{*} E$ and is called the exterior $\lambda$-measure of $A$. This exterior measure satisfies all the standard requirements of a measure function ${ }^{2}$; if $A$ is measurable, in particular if it is closed, we speak of its $\lambda$-measure and denote the latter by $\lambda-m A$. We have further that

(1) The union $V$ of a finite or enumerable sequence of measurable sets $A_{1}, A_{2}, \ldots$ is measurable;

(2) The measure of the sum of a finite or enumerable sequence of measurable sets $A_{1}, A_{2}, \ldots$ no two of which contain a common point is equal to the sum of the measures of those sets, i.e. if $A_{i} A_{j}=0$ for all $i, j$, then

$$
\sum_{i=0}^{\infty}\left(\lambda-m A_{i}\right)=\lambda-m\left(\sum_{i=1}^{\infty} A_{i}\right)
$$

${ }^{1}$ F. Hausdorff, Dimension und äusseres Mass, Math. Annalen, 79 (1919), $157-179$.

2. Carathéodory, Uber das lineare Mass von Punltmengen, Göttingen Nachrichten, 1914, 404-126. 
We define the dimension of a given set $A$ to be $[\lambda(x)]$ if and only if

$$
0<\lambda-m A<\infty
$$

(strict inequality must be preserved).

3. We consider first the non-linear problem. Suppose we take the set $\left\{L_{r}\right\}$ of segments of lines where $L_{r}$ is the segment between the points $(1 / r, 0)$ and $(1 / r, 1)$ on the line $x=1 / r$. Then take a similar set of points $A_{r}$ on each segment-similar in the sense that if, for any $r,(1 / r, y)$ belongs to the set, this holds for all $r$. We make each $A_{r}$ closed and call the similar set on the $y$-axis, the set $A_{0}$. Then we define

$$
A=\sum_{r=0}^{\infty} A_{r}
$$

If now $\lambda-m A_{r}=0$ then obviously $\lambda-m A=0$; and if $\lambda-m A_{r}>0$ then $\lambda-m A=\infty$. Hence the set $A$ is dimensionless.

4. Hausdorff proves that if we define a set $A$ in the interval $(0,1)$ by first omitting a length $1-2 \xi(\xi<1 / 2)$ from the centre of the interval, and then by omitting a length proportional to $1-2 \xi$ from the centres of the two remaining intervals, and so on, in a manner similar to the derivation of the Cantor ternary set then $\lambda-m A=1$ where $\lambda(t)=t^{a}$ and $a=-(\log 2) / \log \xi$. It is easy to prove that if we shrink this set $A$ uniformly into an interval of length $d$ then its measure is $d^{a}$ with the same measure function; this follows directly from the definition of the measure. For the new covering sets will have diameters $\left\{d d_{r}\right\}$ and

$$
\lambda\left(d d_{r}\right)=d^{\alpha} \lambda\left(d_{r}\right)
$$

and we shall have a factor $d^{a}$ coming outside the whole expression for the lower bound, giving our result.

Consider the interval $(1 /(r+1), 1 / r)$ on the $x$-axis. We form in it the set considered in the last paragraph. Call the set $E_{r}$; then its measure will be

$$
\left[\frac{1}{r(r+1)}\right]^{-(\log 2) / \log \xi}
$$

We define, adding in the origin to make the set closed,

$$
E=\sum_{1}^{\infty} E_{r}
$$

then by the theorems of $\S 2, E$ is measurable and

$$
\alpha-m E=\sum_{1}^{\infty}\left(\alpha-m E_{r}\right)
$$


where we denote by $a-m F$ the measure of the set $F$ with measure function $t^{a}$. Hence

$$
a-m E=\sum_{1}^{\infty}\left[\frac{1}{r(r+1)}\right]^{-(\log 2) / \log \xi} .
$$

If we now choose $\xi$ so that

$$
-\frac{\log 2}{\log \xi} \leqq \frac{1}{2}
$$

that is, so that $\xi \leqq \frac{1}{4}$, then $a-m E=\infty$, since the series is divergent.

5. We have now to prove that if we take any function $h(t)$ then either $h-m E=\infty$ or $h-m E=0$. There are two cases to consider.

(i) $\varliminf_{t \rightarrow 0} \frac{h(t)}{l^{a}}=k$ where $0<k \leqq \infty$;

then by the general properties of this measure theory, since $a-m E=\infty$, $h-m E=\infty$ also.

$$
\text { (ii) } \underset{t \rightarrow 0}{\lim _{t \rightarrow 0}} \frac{h\left(t_{r}\right)}{t^{a}}=0 .
$$

We consider this case in detail.

6. We can determine a sequence $t_{1}, t_{2}, t_{3} \ldots$ for which

$$
\frac{h\left(t_{r}\right)}{t_{r}^{a}}<\epsilon, \quad \epsilon>0
$$

and $t_{1}>t_{2}>t_{3}>\ldots$ and $t_{n}$ tends to 0 as $n$ tends to $\infty$. We can cover $E_{r}$ with $2^{n}$ intervals each of length $\xi^{n} / r(r+1)$ where we always have

$$
\frac{2^{n} \xi^{n a}}{[r(r+1)]^{a}}=\frac{1}{[r(r+1)]^{a}}
$$

or $2^{n} \xi^{n a}=1$, for $a=-(\log 2) / \log \xi$ by definition.

We are always able to select a sub-sequence $s_{1}, s_{2} \ldots$ of the sequence $t_{1}, t_{2}, t_{3} \ldots$ such that

$$
\begin{aligned}
& \xi^{n_{1}+1} \leqq s_{1}<\xi^{n_{2}} \\
& \xi^{n_{2}+1} \leqq s_{2}<\xi^{n_{2}} \\
& \cdots \cdots \cdots \cdots \cdots \\
& \xi^{n_{p}+1} \leqq s_{p}<\xi^{n_{*}} \\
& \ldots \ldots \ldots \ldots \ldots
\end{aligned}
$$

The necessity of selecting a sub-sequence arises because more than one $t_{p}$ may lie in between any $\xi^{n}$ and the succeeding $\xi^{n+1}$. 
Then to form the $h$-measure of the set $E$ we must first consider

$$
h(t)-m_{\rho} E_{r}=\text { lower bound } \sum_{U(A, \rho)} h\left(d_{n}\right) .
$$

In this case the spheres $\left\{U_{r}\right\}$ become intervals of equal length and the diameters become their length. Take $\rho=\xi^{n_{p}-1}$; then

$$
h-m_{\rho} E_{r} \leqq 2^{n_{p}+1} h\left(\xi^{n_{p}+1}\right) \leqq 2^{n_{p}+1} h\left(s_{p}\right)
$$

since $h(t)$ increases with $t$ for small $t$. Now by the definition of $s_{r}$

$$
h\left(s_{p}\right)<\epsilon . s_{p}^{a}<\epsilon \xi^{n_{p} a} .
$$

since $t^{\alpha}$ increases with $t$. Hence

$$
h-m_{\rho} E_{r}<\epsilon \cdot 2^{n_{p}+1} \xi^{n_{p^{\alpha}}}=2 \epsilon \cdot\left(2 \xi^{\alpha}\right)^{n}=2 \epsilon .
$$

Hence

$$
h-m E_{r}=\lim _{\rho \rightarrow 0} h-m_{\rho} E_{r}=0
$$

since $\epsilon$ is arbitrary.

The theorems of $\S 2$ give us

$$
h-m E=\sum_{r=1}^{\infty}\left(h-m E_{r}\right)=0 .
$$

No other case can arise since $h(t)$ is always positive and therefore $h-m E$ is zero or infinite for all $h(t)$. Hence the set $E$ has no dimensions.

QUEEN'S UNIVERSITY,

Belfast. 